Monatsschr Kinderheilkd 2017 · 165:780-785 DOI 10.1007/s00112-017-0332-y

Online publiziert: 7. Juli 2017

(c) Der/die Autor(en) 2017. Dieser Artikel ist eine Open-Access-Publikation.

Redaktion

R. Kerbl

E.C. Urban

M. Benesch

CrossMark
E. Nagele · C. Fürschuß · A. Mohapp · K. Wiegele · H. Lackner · C. Urban

Klinische Abteilung für pädiatrische Hämato-Onkologie, Universitätsklinik für Kinder- und Jugendheilkunde, Medizinische Universität Graz, Graz, Österreich

\title{
Von der Diagnose bis zur Transition?
}

\begin{abstract}
Eine onkologische Erkrankung im Kindes- und Jugendalter stellt trotz beeindruckender Überlebenszahlen eine enorme Herausforderung für die PatientInnen und deren Familien dar. Die existenzielle Bedrohung durch die Krankheit, invasive Therapiemaßnahmen und deren Nebenwirkungen sowie der Verlust des sicheren häuslichen und sozialen Umfelds beeinflussen die psychische Befindlichkeit. Eine individuelle psychologische Versorgung ist erforderlich: Ziele und Aufgaben der psychoonkologischen Versorgung haben sich, entsprechend den medizinischen Behandlungsmöglichkeiten, mitund weiterentwickelt. Diese erfolgt heute bedarfsorientiert weit über den Zeitraum der eigentlichen Krebsbehandlung hinaus.
\end{abstract}

\section{Hintergrund}

Seit den Anfängen der Behandlung von onkologischen Erkrankungen im Kindes-/Jugendalter haben sich durch permanente Therapieoptimierung die Prognosen dramatisch verbessert. Multimodale Therapiekonzepte haben sich etabliert [16]. Neben der klassischen „Dreisäulentherapie“ (Kombinationen aus Chemo-, Strahlentherapie und Operationen) mit dem Ziel der kurativen Behandlung [7] kommen innovative und individualisierte Therapiekonzepte zum Einsatz. Massive lebensbedrohliche, akute Nebenwirkungen sowie gravierende
Spät- und Langzeitfolgen sind jedoch häufig der Preis für den Therapieerfolg.

Dadurch wird die psychologische Behandlung in der pädiatrischen Onkologie vor neue Herausforderungen gestellt und eine individualisierte psychosoziale Begleitung oft über viele Lebensphasen hinweg erforderlich [15]. Eine entsprechende Forderung haben Langzeitüberlebende bereits 2006 in der Erice Declaration erhoben [10]. Durch die prädiktive Diagnostik hat die Psychoonkologie außerdem einen „Mehrgenerationenauftrag" bekommen.

Psychosoziale Versorgung als ganzheitliches, interdisziplinäres Behandlungskonzept beruht auf den Prinzipien der Ressourcen-, Familien- und Prozessorientierung und verfolgt 5 übergeordnete Ziele [26]:

- Unterstützung der Krankheitsbewältigung,

- Sicherstellung der Therapie und Kooperation,

- Behandlung spezifischer Symptome,

- sozialrechtliche Beratung und Unterstützung sowie

- Nachsorgeorganisation und Prävention psychosozialer Folgeerscheinungen.

Entsprechend der Leitlinie zur psychosozialen Versorgung in der pädiatrischen Onkologie und Hämatologie sollte prinzipiell jeder betroffenen Familie eine psychosoziale Grundversorgung zuteil werden. Bei erhöhten krankheitsbedingten Primärbelastungen wie ungünstige Prognose, Komplikationen, Rezidiv o. Ä. be- steht die Indikation zu intensivierter psychosozialer Versorgung [26].

\section{Psychosoziale Versorgung im stationären Kontext}

In der Psychoonkologie können die klar umrissene, strukturierende Krisenintervention und die reflektierende, an Ressourcen orientierte supportive Therapie unterschieden werden [24]. Vor allem während der Akuttherapie ist ein prozesshaftes Vorgehen erforderlich. Therapeutische Interventionen orientieren sich stark an den jeweiligen Phasen der onkologischen Behandlung [26]. Auf Basis von Gesprächen und psychologischer Diagnostik werden die psychosozialen Interventionen in den unterschiedlichen Behandlungsphasen laufend angepasst und für die gesamte Familie umgesetzt.

\section{》) Therapeutische Interven- tionen orientieren sich stark an den Phasen der onkologischen Behandlung}

Im Zentrum steht die Kooperation mit dem medizinischen Behandlungsteam. Der Schwerpunkt der Behandlung liegt auf der Förderung der Ressourcen der PatientInnen und ihrer Familie während der Krise der Krankheit, der Therapie sowie - u. U. - des Sterbens, des Todes und der Trauer. Den Kern dazu bildet ein stützendes und informatives Beziehungsangebot [26]. 


\section{Diagnosephase}

Die mit der Diagnose oftmals wahrgenommene Lebensbedrohung stellt einen besonders schwerwiegenden Belastungsfaktor dar [1, 12]. In dieser Phase treten Schock und Angst in den Vordergrund. Es gilt daher, verlässlicher Ansprechpartner zu sein, und über Entlastungsgespräche und Psychoedukation den Diagnoseschock zu überwinden sowie Orientierung zu bieten. Bereitstellen der wichtigsten Informationen sowie psychische Stabilisierung stehen in dieser Phase im Vordergrund und sollen psychischen Folgeerkrankungen vorbeugen. Jahrzehntelange Erfahrung zeigt, dass die Krankheit und evtl. Spätfolgen besser angenommen werden und eine höhere Compliance gegeben ist, je besser es gelungen ist, vertrauensvolle Beziehungen zum gesamten Behandlungsteam aufzubauen. Dazu braucht es konstante AnsprechpartnerInnen sowie ausreichend Raum und Zeit, um auf die Bedürfnisse und Sorgen der Betroffenen eingehen zu können.

\section{》) Bereitstellen der wichtigsten Informationen und psychische Stabilisierung stehen im Vordergrund}

Besonders im Fall der neuen, individualisierten medizinischen Behandlungsstrategien (Target-Therapien) stellt dieser Aspekt aufgrund kürzerer stationärer Aufenthalte und sehr spezifischer Nebenwirkungen eine neue Herausforderung auch für die Psychoonkologie dar.

Eine onkologische bzw. schwere hämatologische Erkrankung eines Kindes betrifft das gesamte Familiensystem. Die Eltern müssen ihrem erkrankten Kind Halt geben und die Folgen der Erkrankung mittragen, sind jedoch selbst durch die lebensbedrohliche Krankheit ihres Kindes in einem äußerst vulnerablen seelischen Zustand und haben auf lange Zeit eine Fülle an psychosozialen Problemen zu bewältigen [9, 17]. So lassen sich in vielen Studien körperliche und psychische Symptome bei engen Angehörigen nachweisen [23]. Schlippe spricht heute von einer „systemischen Familienmedizin“. Dies gilt jedoch nicht nur für die Eltern, sondern auch und insbesondere für die Geschwister krebskranker Kinder, die in der Literatur gern als "Schattenkinder" bezeichnet werden. Bei diesen können häufig negative emotionale Reaktionen, posttraumatische Stresssymptome und eine geringere Lebensqualität im Vergleich zu Gleichaltrigen beobachtet werden [2]. Protektive Faktoren sind offene und ehrliche Kommunikation bereits in der Diagnosephase, Einbezug in den Krankheitsprozess und die PatientInnenbetreuung sowie adäquate Information über Krankheit und Behandlung [26].

\section{Behandlungsphase}

Nachweislich können psychoonkologische Interventionen signifikante Verbesserungen der emotionalen Belastung (Angst, Depression, Stress, Verzweiflung) bzw. eine bedeutsame Steigerung der Lebensqualität und den Aufbau hilfreicher Coping-Strategien bewirken [29]. Psychoedukation zur Förderung des Krankheitsverständnisses und als Vorbereitung auf unterschiedliche diagnostische sowie therapeutische Maßnahmen (Magnetresonanztomographie[MRT]-Untersuchungen, Operationen, Stammzelltransplantationen u. v. m.), Ressourcenaktivierung zur Förderung der Selbstwirksamkeit, Aufbau einer aktiven Bewältigungsstrategie, Ermutigung zum Gefühlsausdruck (wie Trauer, Wut, Angst), Blickverlagerung zu gesunden Anteilen der PatientInnen sowie Stärkung des sozialen Systems (Erziehungs- und Paarberatung) sind besonders wichtige Aufgaben psychologischer Versorgung in dieser Phase.

\section{》) Altersentsprechend wird über Medien mit hohem Aufforderungscharakter eine emotional distanzierte Auseinandersetzung ermöglicht}

Den Sinn der erforderlichen Behandlungsmaßnahmen zu verstehen, ist notwendig, um eine Vertrauensbasis mit dem Patienten/der Patientin herzustellen und ihn/sie aktiv in den therapeutischen Prozess einzubeziehen.

Für eine altersadäquate Aufklärung über die Erkrankung und deren Behandlung ist es im direkten Gespräch besonders wichtig, auch auf nonverbale Signale der Kinder zu achten und ihre Grenzen $\mathrm{zu}$ akzeptieren. Über Medien wie Bücher, Spiele, Geschichten, Zeichnungen, Filme etc. ist eine emotional distanzierte Auseinandersetzung mit den Themen Krankheit, Nebenwirkungen der medizinischen Behandlung, Untersuchungen, pflegerische Maßnahmen etc. möglich. Durch die emotionale Trennung von der eigenen Person und den Einsatz von Materialien mit hohem Aufforderungscharakter wird Angst reduziert und Neugier und Offenheit dem Thema gegenüber gefördert.

\section{》) Psychologische Interventio- nen bewirken Verbesserungen der emotionalen Belastung}

Ressourcen sind für die Betroffenen von größter Bedeutung, um mit der Krankheitssituation umzugehen und damit ein weiteres zentrales Thema der psychoonkologischen Behandlung. Krankheitsverarbeitung ist ein aktiver Prozess, der sowohl die Nutzung vorhandener als auch die Entwicklung neuer Verhaltensweisen und die Erschließung zusätzlicher Ressourcen erfasst. Als protektiv wirksame Faktoren für die kindliche Entwicklung und die Verarbeitung von kritischen Lebensereignissen werden neben individuellen Ressourcen, wie positiver Selbstwahrnehmung, Kohärenzsinn und Optimismus soziale Ressourcen, wie die soziale Unterstützung inner- und außerhalb der Familie sowie Merkmale eines förderlichen Erziehungsklimas benannt [5].

Die Wirksamkeit psychologischer Interventionen zur Reduktion von Schmerz und chemotherapiebedingten Nebenwirkungen ist ebenfalls wissenschaftlich gesichert [29, 32] Psychologische Schmerzbehandlung setzt auf den Ebenen der Schmerzwahrnehmung und -bewertung an. Ihr Ziel ist nicht in erster Linie absolute Schmerzfreiheit, 
sondern die Minimierung der Belastung durch die Schmerzsituation sowie das Vermeiden von Traumatisierung und Schmerzsensibilisierung. Die Erarbeitung von Bewältigungs- und Kontrollmechanismen soll schmerzspezifische Einstellungen beeinflussen und ein adäquates Schmerzverhalten fördern. Als erfolgreiche psychologische Behandlungskonzepte werden diesbezüglich u. a. Psychoedukation, kognitive Uminterpretation, Selbstinstruktion, Entspannungsverfahren, Aktivitätsaufbau, Aufmerksamkeitslenkung, Imagination und Hypnose eingesetzt.

Darüber hinaus werden psychodiagnostische Verfahren zur Früherkennung von Defiziten v. a. im Bereich der Konzentrations-, Wahrnehmungs- und Gedächtnisleistungen durchgeführt und bei Bedarf spezielle Trainings angeboten.

Gegen Ende der Behandlungsphase werden die vollständige und lückenlose Übergabe in die Nachsorgeambulanz sowie die Sicherung möglicher Nachsorgeangebote außerhalb der Klinik angestrebt.

\section{Psychosoziale Nachsorge}

Krankheitsbezogene Erfahrungen und Risiken möglichst gut $\mathrm{zu}$ bewältigen und bestmöglich in die eigene Biografie $\mathrm{zu}$ integrieren, sind langfristige Ziele im Leben pädiatrisch onkologischer $\mathrm{Pa}$ tientInnen. Geheilt zu sein, bedeutet nicht, gesund zu sein. Aus den neuesten Datenanalysen der Childhood Cancer Survivor Study geht u. a. hervor, dass sich die 15-Jahres-Sterblichkeit von 12,4\% der PatientInnnen, die in den 1970erJahren behandelt wurden, bei Kindern/ Jugendlichen in den 1990er-Jahren auf $6,0 \%$ halbiert hat [3]. Trotz optimierter und verbesserter Therapiemaßnahmen bedingen die Erkrankung und die intensiven Behandlungsmaßnahmen aber nach wie vor bei vielen jungen Menschen z. T. schwerwiegende körperliche Spätfolgen. Ein Drittel der Langzeitüberlebenden kann nach onkologischen Erkrankungen im Kindesalter in ein normales Alltagsleben zurückkehren. Ein Drittel der PatientInnen leidet an minimalen Spätfolgen, das restliche Drittel an gravierenden Spätfolgen, die eine

Monatsschr Kinderheilkd 2017 · 165:780-785 DOI 10.1007/s00112-017-0332-y

(c) Der/die Autor(en) 2017. Dieser Artikel ist eine Open-Access-Publikation.

\section{E. Nagele · C. Fürschuß · A. Mohapp · K. Wiegele · H. Lackner · C. Urban}

\section{Psychosoziale Versorgung pädiatrisch-onkologischer PatientInnen. Von der Diagnose bis zur Transition?}

\section{Zusammenfassung}

Eine Krebserkrankung und -behandlung im Kindesalter hat gravierende Auswirkungen auf Lebenskontext und Lebensperspektive der PatientInnen und ihrer Angehörigen. Entsprechend hohe gesundheitspolitische Relevanz hat eine adäquate psychosoziale Versorgung im Sinne der Prävention psychischer und sozialer Langzeitfolgen sowie der Förderung individuell-adaptiver Prozesse, die entscheidend sind für das Gelingen des Lebens während und nach der Erkrankung. Ziel der Arbeit ist eine zusammenfassende Übersicht über etablierte Versorgungskonzepte und aktuelle Herausforderungen in der pädiatrischen Psychoonkologie. Internationale Leitlinien bilden die Basis einer bedarfsgerechten, evidenzbasierten pädiatrisch-psychoonkologischen Behandlung. Diese beeinflusst nachweislich wesentliche psychosoziale Variablen bei Betroffenen und deren Angehörigen. Basis aller Konzepte sind neben der Familienorientierung die Stärkung von Ressourcen sowie eine prozessorientierte, multiprofessionelle und supportive
Therapie. Im Sinne eines ganzheitlichen Behandlungskonzeptes in der pädiatrischen Hämatoonkologie leistet die psychosoziale Versorgung wichtige Beiträge zu Krankheitsverarbeitung, Compliance, Lebensqualität, PatientInnenzufriedenheit sowie zu Gesundheitsförderung und Reintegration. Grundlage bildet eine bedarfsgerechte Betreuung und Begleitung ab Diagnosestellung während der Akuttherapie sowie nach Therapieende. Generationenübergreifende onkologische Erkrankungen erfordern zudem spezielle psychoedukative und therapeutische Angebote für die ganze Familie. Strukturund Rahmenbedingungen sollen geschaffen werden, um psychosoziale Behandlungs-, Nachsorge- und Überleitungskonzepte weitreichend zu implementieren.

Schlüsselwörter "Survivors" · Lebensqualität · Behandlungsstandard - Case Management . Nachsorge

\section{Psychosocial care of pediatric oncology patients. From the diagnosis up to transition?}

Abstract

Cancer and its treatment in childhood have serious effects not only on the context of life but also on the perspectives for life of patients and their relatives. An appropriate psychosocial care is highly relevant for healthrelated political issues by preventing psychological and social long-term effects. Adaptive activities for individuals are promoted, which are important for a successful life during and after the disease. The aim of this article is to give a comprehensive overview of wellestablished concepts in psychosocial care and current challenges in pediatric psychooncology. International guidelines form the basis of a needs-oriented, evidence-based psycho-oncological treatment. This treatment definitely has positive effects on specific psychosocial variables in patients and their family members. The basic elements of all theoretical concepts are the reinforcement of resources and a treatment-related multiprofessional supportive therapy, in addition to family orientation. Psychosocial care makes important contributions to processing of the disease, compliance and an increase in quality of life, patient satisfaction as well as health promotion and reintegration in the sense of a holistic treatment concept in pediatric oncology and hematology. The foundations are needs-oriented care and accompaniment from the initial diagnosis, during the acute treatment and also after termination of treatment. Cross-generational oncological diseases require specific psychoeducational and therapeutic options for the whole family. Structural and general framework conditions must be achieved for a sustainable implementation of psychosocial care during treatment, follow-up and transition.

\section{Keywords}

Survivors - Quality of life - Standard of care . Case management $\cdot$ Aftercare 
langfristige, multidisziplinäre Betreuung auch im Erwachsenenalter erfordern [13]. Der Anteil an PatientInnen mit Spätfolgen wird in ähnlichen Arbeiten auf $60-75 \%$ der Betroffenen geschätzt [18, 20, 27].

Laut der Arbeitsgruppe Spätfolgen Late Effects Surveillance System (LESS) werden über $90 \%$ der "survivors“ mit zunehmendem Lebensalter neu diagnostizierte Folgeerkrankungen erleiden [14]. Pädiatrisch-onkologische PatientInnen gehören zudem zur Risiko- bzw. Hochrisikogruppe für die Entwicklung eines Zweitmalignoms [6, 8]; dreißig Jahre nach Erstdiagnose beträgt das Risiko durchschnittlich etwa $6,6 \%$ für alle Krebsarten [11]. Diese Tatsache kann eine erhebliche psychische Belastung darstellen [31].

\section{》) Geheilt zu sein, bedeutet nicht, gesund zu sein}

Des Weiteren kann eine Krebserkrankung im Kindes- und Jugendalter massive psychosoziale Langzeitfolgen bedingen. Krankheitsbezogene Ängste, affektive Störungen, Schwierigkeiten im sozialen Bereich, Hürden in Ausbildung und Beruf sowie neuropsychologische Spätfolgen [25] zählen zu den häufigsten.

Die Langzeitanalysen der „Swiss Childhood Cancer Survivor Studie“ haben u. a. gezeigt, dass nicht nur die Anzahl von Survivors mit klinisch relevanten psychischen Belastungen im Studienverlauf zunimmt, sondern auch die Intensität dieser Belastungen [22].

Psychosoziale Nachsorge beginnt als Teil eines multidisziplinären Nachsorgeangebots spätestens mit dem Übergang von der Akuttherapie in die Nachsorge.

Die erste Zeit nach der Akutbehandlung ist oft gekennzeichnet von großen Erwartungshaltungen, aber auch Ängsten, Erschöpfung und den Herausforderungen des „neuen Alltags“. Ein bedarfsorientiertes und individuelles Angebot wird entwickelt (individuelles psychosoziales Follow-up/Case Management). Dazu gehören neben Beratung und Psychoedukation häufig auch Psychodiagnostik sowie die Einleitung bzw. Vermittlung von Maßnahmen zu För- derung und/oder Therapie, z. B. bei neurokognitiven Krankheitsfolgen und Entwicklungsauffälligkeiten oder Psychotherapiebedarf. Information und Hilfestellung zur Auswahl passender Rehabilitationsmaßnahmen (familienorientierte Rehabilitation, Neuro-, Jugendrehabilitation, Nachsorgeangebote der Österreichischen Kinderkrebshilfe) sowie Beratungs- und Behandlungsangebote $\mathrm{zu}$ sozialen, schulischen und beruflichen Integrationsmaßnahmen spielen ebenso eine wesentliche Rolle wie das interdisziplinäre Erarbeiten eines individuellen Reintegrationsplans.

Mit dem Fortschritt der medizinischen Therapiemaßnahmen haben sich auch die psychosozialen Standards verändert [30]. Nachsorgekonzepte haben sich zu teils generationenübergreifenden Vorsorgekonzepten weiterentwickelt.

Im Jahr 2003 wurde am Univ.-Klinikum Graz ein Therapieoptimierungsprotokoll für Retinoblastome erstellt, das für PatientInnen mit erblicher Erkrankung eine umfassende Nachsorge bis ins Erwachsenenalter empfiehlt [28]. Bei dieser PatientInnengruppe besteht die Neigung, im weiteren Leben andere bösartige Tumoren zu entwickeln, und ein etwa 50 \%iges Risiko, diese Veranlagung an ihre Nachkommen zu vererben [19]. Das Leben mit der Angst vor einem weiteren Malignom, regelmäßige augenärztliche und onkologische Kontrollen sowie massive Auswirkungen auf individuelle Lebenskonzepte wie Partnerschaft und Familienplanung sind Stressoren, denen diese PatientInnen lebenslang ausgesetzt sind.

\section{》) Fortführung der psycho- sozialen Unterstützung durch Nachsorge darf nicht nur eine Empfehlung bleiben}

Generell treten Sekundärmalignome bei pädiatrisch-onkologischen PatientInnen 10-mal häufiger auf als Krebserkrankungen in altersentsprechenden Gruppen [7].Zudem werden immer mehr Krebserkrankungen im Kindes- und Jugendalter auf eine Krebsdisposition zurückgeführt [21].
Im Rahmen der Erwachsenensprechstunde für Survivors in Graz wurde ein Pilotprojekt zur individuell angepassten psychoonkologischen Prävention (primär für Familien mit hereditärem Tumorprädispositionssyndrom, tertiär für ehemalige KrebspatientInnen) realisiert. Es wurde ein spezielles Informationsund Stressmanagementprogramm entwickelt, das sich an aktuellen Ergebnissen aus verschiedenen interdisziplinären Forschungsbereichen (z. B. Psychoneuroimmunologie, Salutogeneseforschung, Hirnforschung) orientiert, um die Resilienz der Survivors zu steigern und damit positiven Einfluss auf ihr Wohlbefinden und ihre Gesundheit zu nehmen. Dieses Angebot ist jedoch weder österreichnoch europaweit etabliert. Zurecht fordern Survivors umfangreiche Aufklärung über mögliche Spätfolgen sowie europaweite Standards in den Nachsorge-, Vorsorge- und Transitionsprogrammen [25]. Die Fortführung der psychosozialen Unterstützung durch multidisziplinäre Nachsorge und die Erfassung von Risikofaktoren im Anpassungsprozess darf nicht nur eine Empfehlung bleiben [26].

\section{Diskussion}

Diese Arbeit bietet einen Überblick über etablierte psychosoziale Versorgungskonzepte auf wissenschaftlicher Basis. Gemeinsames Ziel aller Interventionen ist die bedarfsgerechte, systemorientierte, supportive Betreuung mit dem Bestreben, erkrankungs- und behandlungsbedingte Belastungen bestmöglich zu bewältigen und psychosozialen Langzeitfolgen vorzubeugen.

Aktuelle Entwicklungen in der medizinischen Behandlung mit den dadurch auf die psychosoziale Versorgung zukommenden neuen Herausforderungen und Aufgaben werden angesprochen.

Die stetige Weiterentwicklung der Therapien (Target-Therapie) führt in vielen Fällen zu verkürzten stationären Aufenthalten und weniger bzw. anderen Nebenwirkungen, was Auswirkungen auf Krankheitseinsicht und Compliance haben kann. Die psychosoziale Versorgung wird hier neue Wege finden müssen, um auf der Basis konstanter Beziehungsangebote die PatientInnen 
im Therapieverlauf weiterhin optimal unterstützen zu können. Andererseits werden bereits jetzt Knochenmark- und Stammzelltransplantationen vermehrt auf nichtonkologische Erkrankungen ausgeweitet. Ein vergleichsweise geringes Krankheitsgefühl und wenige Einschränkungen vor der Transplantation, kurze Vorlaufzeiten zur Vorbereitung der PatientInnen, eine intensive Therapie mit oft massiven Nebenwirkungen und langwierige, die Lebensqualität manchmal über Monate oder Jahre beeinträchtigende Langzeitfolgen stellen sowohl die PatientInnen als auch die (psychosozialen) BehandlerInnen vor neue Herausforderungen.

Auch die prädiktive Diagnostik wird zunehmend weiterentwickelt, ca. $10 \%$ aller Krebserkrankungen sind heute auf eine erbliche Tumordisposition zurückzuführen. Dies eröffnet der Medizin eine völlig neue Dimension der Krankheitsprävention, in der die psychosoziale Versorgung ebenfalls eine tragende Rolle spielen wird [4]. Doch die Identifikation einer Person mit erhöhtem Krebsrisiko ist nur dann sinnvoll, wenn sie zur Verbesserung der Lebenserwartung und der Lebensqualität führt. Es ist eine Herausforderung für die Psychoonkologie als Teil der präventiven Onkologie, jene klinischen Managementstrategien für die betroffenen PatientInnen/Familien mitzuentwickeln, die nach der Diagnosestellung zu einer Anpassung der Lebensgewohnheiten führen können, um dadurch eine Risikoverringerung zu erreichen.

\section{Fazit für die Praxis}

- Psychologische Behandlung mit ihren nachweislich wirksamen Methoden soll ausreichend in die pädiatrischonkologische Standardversorgung integriert und jeder Familie zur Verfügung gestellt werden.

- Innovative und individualisierte medizinische Therapieoptionen bedürfen der Entwicklung eines adaptierten psychologischen Versorgungskonzeptes.

- Psychosoziales Follow-up/Case Management der Survivors und ihrer Angehörigen soll kontinuierlich sein und ein Leben lang angeboten werden können.

- Die Nachsorge soll spezialisiert sein und individuelle Vorsorgemaßnahmen umfassen, um eine erfolgreiche Transition und letztlich gesellschaftliche Partizipation zu ermöglichen.

- Translationale Forschungsprojekte mit psychosozialen Schwerpunkten sollen vermehrt umgesetzt werden. - Europäische (Nachsorge-)Leitlinien sollen laufend auf aktueller Studienbasis erarbeitet werden.

\section{Korrespondenzadresse}

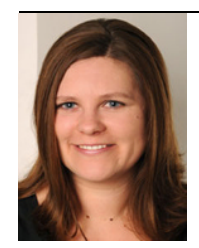

MMag. Dr. E. Nagele

Klinische Abteilung für pädiatrische Hämato-Onkologie, Universitätsklinik für Kinderund Jugendheilkunde,

Medizinische Universität Graz Auenbruggerplatz 34/2, 8036 Graz, Österreich eva.nagele@medunigraz.at

Open access funding provided by Medical University of Graz.

\section{Einhaltung ethischer Richtlinien}

Interessenkonflikt. E. Nagele, C. Fürschuß, A. Mohapp, K. Wiegele, H. Lackner und C. Urban geben an, dass kein Interessenkonflikt besteht.

Dieser Beitrag beinhaltet keine von den Autoren durchgeführten Studien an Menschen oder Tieren.

Open Access. Dieser Artikel wird unter der Creative Commons Namensnennung 4.0 International Lizenz (http://creativecommons.org/licenses/by/4.0/deed. de) veröffentlicht, welche die Nutzung, Vervielfältigung, Bearbeitung, Verbreitung und Wiedergabe in jeglichem Medium und Format erlaubt, sofern Sie den/die ursprünglichen Autor(en) und die Quelle ordnungsgemäßnennen, einen Linkzur Creative Commons Lizenz beifügen und angeben, ob Änderungen vorgenommen wurden.

\section{Literatur}

1. Al-GamalE, Long T(2016) Health-related quality of life and its association with self-esteem and fatigue among children diagnosed with cancer. J Clin Nurs 25:3391-3399

2. Alderfer MA, Long KA, Lown EA et al (2010) Psychosocial adjustment of siblings of children with cancer: a systematic review. Psychooncology 19:789-805

3. Armstrong GT, Chen Y, Yasui $Y$ et al (2016) Reduction in late mortality among 5-year survivors of childhood cancer. N Engl J Med 374:833-842
4. Bachmann K (1998) Richtlinien zur Diagnostik der genetischen Disposition für Krebserkrankungen. Dtsch Arztebl 95:A-1396

5. Bettge S, Ravens-Sieberer U (2003) Protective factors for mental health of children and adolescent empirical results validating a concept. Gesundheitswesen 65:167-172

6. Carretier J, Boyle H, Duval S et al (2016) A review of health behaviors in childhood and adolescent cancer survivors: toward prevention of second primary cancer. J Adolesc Young Adult Oncol 5:78-90

7. Creutzig U, Henze G, Bielack S et al (2003) Krebserkrankungen bei Kindern, Erfolg durch einheitliche Therapiekonzepte seit 25 Jahren. Dtsch Arztebl 100:842-852

8. Friedman DN, Chou JF, Oeffinger KC et al (2016) Chronic medical conditions in adult survivors of retinoblastoma: results of the retinoblastoma survivor study. Cancer 122:773-781

9. Goldbeck L (2001) Parental coping with the diagnosis of childhood cancer: gender effects, dissimilarity within couples, and quality of life. Psychooncology 10:325-335

10. Haupt R, Spinetta JJ, Ban I et al (2007) Long term survivors of childhood cancer: cure and care. The Erice statement. Eur J Cancer 43:1778-1780

11. Kaatsch P, Grabow D, Spix C (2016) German childhood cancer registry - annual report 2016 (1980-2015). Insitute of Medical Biostatistics, Epidemiology and Informatics (IMBEI) at the University Medical Center of the Johannes Gutenberg University Mainz, Mainz

12. Kazak AE, Noll RB (2015) The integration of psychology in pediatric oncology research and practice: collaboration to improve care and outcomes for children and families. Am Psychol 70:146-158

13. Lackner H, Moser A, Sovinz P et al (2007) Longterm care of young adults surviving childhood cancer - Where do we go? Wien Klin Wochenschr 119:361-364

14. Langer T (2015) Langzeitnachsorge und -beobachtung nach einer Krebserkrankung im Kindes- und Jugendalter. Monatsschr Kinderheilkd 163:1177-1178

15. Langeveld NE, Stam H, Grootenhuis MA et al (2002) Quality of life in young adult survivors of childhood cancer. Support Care Cancer 10:579-600

16. Längler A, Herold R, Kaatsch $P$ et al (2008) Pädiatrische Onkologie - Heutiger Stand der Kinderonkologie. Med Welt 59:99-104

17. Ljungman L, Cernvall M, Gronqvist $\mathrm{H}$ et al (2014) Long-term positive and negative psychological late effects for parents of childhood cancer survivors: a systematic review. PLOSONE 9:e103340

18. Oeffinger KC, Mertens AC, Sklar CA et al (2006) Chronic health conditions in adult survivors of childhood cancer. NEngl J Med 355:1572-1582

19. Peck-Himmel J (2011) Weiß sehen. Augenkrebs bei Kindern frühzeitig erkennen. Sonne 9-11. doi:10. 1007/s00112-017-0332-y

20. Pogany L, Barr RD, Shaw A et al (2006) Health status in survivors of cancer in childhood and adolescence. Qual Life Res 15:143-157

21. Ripperger T, Bielack SS, Borkhardt A et al (2017) Childhood cancer predisposition syndromesA concise review and recommendations by the cancer predisposition working group of the Society for Pediatric Oncology and Hematology. Am J Med Genet A 173:1017-1037

22. Rueegg CS, Gianinazzi ME, Rischewski J et al (2013) Health-related quality of life in survivors 
of childhood cancer: the role of chronic health problems. J Cancer Surviv 7:511-522

23. Schlippe A (2003) Chronische Krankheit im Kontext sozialer Systeme. systhema 1/2003:20-37

24. Schlömer-Doll U (2006) Psychoonkologische Begleitung. Von der Diagnose bis zum Tod. Dtsch Arztebl 2:73-75

25. Schneider C (2015) Psychosoziale Nachsorge in der pädiatrischen Onkologie. Fakultät für Psychologie Universität Wien, Wien

26. Schröder HM, Lilienthal S, Schreiber-Gollwitzer BM et al (2013) Psychosoziale Versorgung in der Pädiatrischen Onkologie und Hämatologie. In: AWMF-Leitlinie Register Nr. 025/002 - Entwicklungsstufe 3 Psychosoziale Arbeitsgemeinschaft in der pädiatrischen Onkologie und Hämatologie (PSAPOH). AG in der Gesellschaft für pädiatrische Onkologie und Hämatologie (GPOH). Arbeitsgemeinschaft der Wissenschaftlichen Medizinischen Fachgesellschaften (AWMF), Lübeck

27. Skinner R, Wallace WH, Levitt G (2007) Long-term follow-up of children treated for cancer: Why is it necessary, by whom, where and how? Arch Dis Child 92:257-260

28. Sovinz P, Urban C, Lackner $\mathrm{H}$ et al (2006) Retinoblastoma: a proposal for a multimodal treatment concept for intraocular retinoblastoma in Austria. Wien Klin Wochenschr 118:22-30

29. Tschuschke V (2011) Psychoonkologie - Psychologische Aspekte der Entstehung und Bewältigung von Krebs. Schattauer, Stuttgart

30. Urban CE, Mutz ID (1978) Psychological management of children with malignancies and their families (author's transl). Padiatr Padol 13:137-144

31. Wang R, Syed IA, Nathan PC et al (2015) Exploring cancer worry in adolescent and young adult survivors of childhood cancers. J Adolesc Young Adult Oncol 4:192-199

32. Weis J, HecklU, Brocai Detal (2006) Psychoedukation mit Krebspatienten - Therapiemanual für eine strukturierte Gruppenintervention. Schattauer, Stuttgart

\section{Gerhard Jorch / Dirk Schlüter (Hrsg.) Fetoneonatale Infektiologie}

Stuttgart: Georg Thieme Verlag 2017, 1. Aufl., 248 S., 76 Abb., (ISBN: 978-3-13-174891-1), Hardcover 179,99 EUR

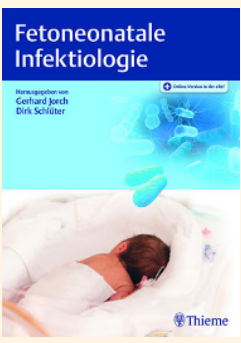

An sich ist es ja verwunderlich, dass es immer noch "Lücken" gibt für neue Buchtitel. Die soeben erschienene "Fetoneonatale Infektiologie" füllt eine solche. Der

Direktor einer Universitätskinderklinik mit Perinatalzentrum (G. Jorch) und der Direktor eines universitären mikrobiologischen Instituts (D. Schlüter) haben mit 16 weiteren Autoren ein 248 Seiten umfassendes Werk vorgelegt, das sich v.a. an medizinisches Personal in Perinatalzentren wendet.

Das Buch ist in 7 Kapitel untergliedert, inhaltlich lassen sich de facto 3 Abschnitte abgrenzen. Im 1.Teil (Kapitel 1-5) werden auf 84 Seiten eher "allgemeine“ Feststellungen zur fetoneonatalen Infektiologie getroffen. Themen sind u.a. Entwicklung des Immunsystems, Infektionszeichen und -marker, therapeutische Grundsätze, Hygienemaßnahmen. Der 2.Teil (Kapitel 6) beschreibt auf 79 Seiten relevante spezifische Infektionen, die alphabetisch gereiht sind - von A wie "Aspergillose" bis Z wie Zikavirus. Lediglich die "Influenza" ist an etwas unlogische Stelle zwischen "Tuberkulose" und "Zikavirus" verrutscht. Der 3. Teil widmet sich schließlich unter der Überschrift „Prävention, Diagnostik und Therapie während der Schwangerschaft und Geburt" einigen ausgewählten Infektionen (u.a. CMV, Hepatitis, Herpesvirus, HIV, Streptokokken B). Als Leser stellt man sich die Frage warum 2. und 3. Teil nicht zu einem Kapitel zusammengefasst wurden, dies hätte eventuell eine etwas bessere Übersichtlichkeit ergeben.

Ansonsten sind die einzelnen Erkrankungen umfassend und gut verständlich beschrieben, wobei sich die Angaben nicht ausschließlich auf die Perinatalzeit beschränken. Dies ermöglicht eine umfassende Sicht auf die beschriebenen Erkrankungen. Insbesondere der 2. Teil gibt durch zahlreiche "Merke" praxisrelevante Tipps, und ergänzende
Fallbeispiele unterstützen das Krankeitsverständnis. Die insgesamt 76 Abbildungen sind etwas ungleichmäßig auf die einzelnen Erkrankungen verteilt, manche davon dürften älteren Datums sein.

Positiv festzuhalten ist, dass das Buch kaum Fragen zur Thematik "Fetoneonatale Infektiologie" offen lässt. Neben Tabellen zur antibiotischen Therapie und Beschreibung von „Problemkeimen“ werden z.B. auch Grundsätze der Hygiene und der Frauenmilchspende dargestellt. Dabei orientiert sich das Buch u.a. am US-amerikanischen Red Book, dem Deutschen Epidemiologischen Bulletin sowie diversen AWMF-Leitlinien.

Zusammenfassend handelt es sich um ein Buch, das an keinem deutschsprachigen Perinatalzentrum fehlen sollte, zumindest ein Exemplar sollte dort in der Bibliothek stehen. Das Buch eignet sich sowohl als Lernunterlage für in Ausbildung Befindliche als auch als Nachschlagewerk bei spezifischen Fragestellungen.

Neben der gebundenen Ausgabe ist der Titel auch als eBook erhältlich. Insbesondere für die Jüngeren dürfte auch die Verfügbarkeit mittels QR-Code oder Link und Zugangscode interessant sein, wobei der elektronische Zugang mit dem Kauf des Buches ohne Zusatzkosten ermöglicht wird. Die 179,99€ sind auch aus diesem Grund eine sinnvolle Investition .....

R. Kerbl, Leoben 